TRANSACTIONS OF THE

AMERICAN MATHEMATICAL SOCIETY

Volume 147, February 1970

\title{
CONJUGATE FUNCTIONS FOR LAGUERRE EXPANSIONS
}

\author{
BY \\ BENJAMIN MUCKENHOUPT $\left({ }^{1}\right)$
}

1. Introduction. The purpose of this paper is to study a natural conjugate function for a function with a Laguerre polynomial expansion. Theorems similar to the well-known ones for conjugate Fourier expansions and theorems in [2] and [4] for Gegenbauer and Hermite expansions are proved. As in the other cases, it is proved that the mapping from a function to its conjugate function or its conjugate Poisson integral is a bounded operator in $L^{p}$ with the appropriate measure for $1<p<\infty$. The conjugate Poisson integral converges in $L^{p}$ norm and pointwise almost everywhere to the conjugate function. Substitute results for the case of $L^{1}$ are also proved. This leads to the possibility of an $H^{p}$ theory for Laguerre expansions and analogues of the other theorems concerning the classical conjugate function.

If $f(y)$ is in $L^{1}\left(e^{-y} y^{\alpha}\right)$ on $(0, \infty)$ and $\alpha>-1$, the Poisson integral defined in [3] will be used and in $\S 2$ a definition of the conjugate Poisson integral, $f^{x}(x, y)$, will be given for $x>0$. Notation and estimates are given in $\S 2$ that are needed in the other sections.

In $\S 3$ the existence of $\tilde{f}(x, y)$ for any $f(y)$ in $L^{1}\left(e^{-y} y^{\alpha}\right)$ is proved. If $f(y)$ has Laguerre expansion $\sum a_{n} L_{n}^{\alpha}(y)$, then, as shown in [2], for each $x>0, f(x, y)$ has Laguerre expansion $\sum a_{n} \exp (-x \sqrt{ } n) L_{n}^{\alpha}(y)$. In $\S 3, \tilde{f}(x, y) / \sqrt{ } y$ is shown to have Laguerre expansion

$$
\sum \frac{-a_{n} \exp (-x \sqrt{ } n) L_{n+1}^{\alpha+1}(y)}{\sqrt{ } n}
$$

As in [2] and [3], defining $f(x, y)$ to be $\sqrt{ } y$ times the sum of this series would have been unsatisfactory since the series may diverge for all $y$ for some $x>0$ and $f(y)$ in $L^{p}\left(e^{-y} y^{\alpha}\right), 1 \leqq p<2$.

It was shown in [3] that for $x>0$

$$
\frac{\partial^{2} f(x, y)}{\partial x^{2}}+e^{y} y^{-\alpha} \frac{\partial}{\partial y}\left[e^{-y} y^{\alpha+1} \frac{\partial f(x, y)}{\partial y}\right]=0 .
$$

It is shown in $\S 3$ that

$$
\frac{\partial^{2} \tilde{f}(x, y)}{\partial x^{2}}+(\sqrt{ } y) \frac{\partial}{\partial y}\left(e^{y} y^{-\alpha} \frac{\partial}{\partial y}\left[e^{-y} y^{\alpha+1 / 2} \tilde{f}(x, y)\right]\right)=0
$$

Received by the editors July $15,1967$.

(1) Supported in part by NSF grants GP4219 and GP7539.

Copyright (C) 1970, American Mathematical Society 
and that the analogues of the Cauchy Riemann equations

$$
\frac{\partial f(x, y)}{\partial x}=e^{y} y^{-\alpha} \frac{\partial}{\partial y}\left[e^{-y} y^{\alpha+1 / 2} \tilde{f}(x, y)\right]
$$

and

$$
\frac{\partial f(x, y)}{\partial y}=\frac{-1}{\sqrt{ } y} \frac{\partial \tilde{f}(x, y)}{\partial x}
$$

are valid for $x>0$. Equation (1.4) can also be written in the form

$$
\frac{\partial f(x, y)}{\partial y}=-e^{y} y^{-\alpha-1} \frac{\partial}{\partial x}\left[e^{-y} y^{\alpha+1 / 2} \tilde{f}(x, y)\right] \text {. }
$$

Because of (1.3) and (1.5), it might seem more natural to define $e^{-y} y^{\alpha+1 / 2} \tilde{f}(x, y)$ as the conjugate integral. Since the norm inequalities are true for $f(x, y)$, however, it is the conjugate integral that will be used.

In $\$ 4$ a lemma about the conjugate Poisson kernel is stated so that general theorems proved in [2] and [3] can be applied to this case. Unfortunately, the expressions that arise present considerable technical difficulty since they are complicated and include Bessel functions. The principal term that arises is, however, almost the same as the kernel considered in [2] for the Hermite case. This is the hardest part to estimate and use can be made of the results in [2]. The other terms are numerous; a description of how they can all be estimated is given in $\S \S 5$, 6 and 7.

Finally in $\S 8$ the lemma is used to show that $f(x, y)$ is dominated by a Hardy max function plus a sup of singular integrals. This is then used to prove the various norm and convergence properties in the same way as that was done in [2]. The conjugate function, $\tilde{f}(y)$, is defined as $\lim _{x \rightarrow 0^{+}} \tilde{f}(x, y)$ and facts about it are proved.

2. Definitions and notation. It will be assumed throughout this paper that $\alpha$ is a fixed number greater than -1 , that $y$ and $z$ are nonnegative and $0<r<1$. $C$ will denote a constant depending only on $\alpha$ but not necessarily the same at each occurrence. If $f(y)$ is in $L^{1}\left(e^{-y} y^{\alpha}\right)$ on $(0, \infty)$, the Poisson integral, $f(x, y)$, will be defined as it was in (4.21) of [3] as

$$
f(x, y)=\int_{0}^{\infty} P f(z) e^{-z} z^{\alpha} d z
$$

where

$$
P=\int_{0}^{1} U(x, r) \frac{\exp \left(\frac{-r(y+z)}{1-r}\right) I_{\alpha}\left(\frac{2 \sqrt{ }(r y z)}{1-r}\right)}{(1-r)(r y z)^{\alpha / 2}} d r
$$

and

$$
U(x, r)=\frac{x \exp \left(\frac{x^{2}}{4 \log r}\right)}{2 \pi^{1 / 2} r(-\log r)^{3 / 2}}
$$

and $I_{\alpha}(x)=i^{-\alpha} J_{\alpha}(i x)$ is the usual Bessel function of an imaginary argument. 
Using (5.1.15), p. 101 of [6] shows that

$$
P=\int_{0}^{1} U(x, r) \sum_{n=0}^{\infty} \frac{r^{n} L_{n}^{\alpha}(y) L_{n}^{\alpha}(z)}{\Gamma(\alpha+1)\left(\begin{array}{c}
n+\alpha \\
n
\end{array}\right)} d r
$$

in fact (2.2) was originally obtained from this. Formula (4.20) of [3] is

$$
\int_{0}^{1} U(x, r) r^{n} d r=\exp (-x \sqrt{ } n) .
$$

Now, (7.6.11), p. 176 of [6] shows that for a fixed $C>0$

$$
L_{n}^{\alpha}(x)=O\left(n^{\alpha+1 / 4}\right), \quad 0 \leqq x \leqq C .
$$

Because of (2.5), (2.6) and the dominated convergence theorem, the summation and integration can be interchanged in (2.4). This and (2.5) then gives

$$
P=\sum_{n=0}^{\infty} \frac{\exp (-x \sqrt{ } n) L_{n}^{\alpha}(y) L_{n}^{\alpha}(z)}{\Gamma(\alpha+1)\left(\begin{array}{c}
n+\alpha \\
n
\end{array}\right)}, \quad x>0 .
$$

The following facts about $I_{\alpha}$ will be used. From [7, p. 77]

$$
I_{\alpha}(z)=\left(\frac{1}{2} z\right)^{\alpha} / \Gamma(\alpha+1)+O\left(z^{\alpha+2}\right), \quad 0<z \leqq 1,
$$

and from [7, p. 203],

$$
I_{\alpha}(z)=\frac{e^{z}}{\sqrt{ }(2 \pi z)}\left[1+O\left(z^{-1}\right)\right], \quad 1 \leqq z .
$$

To estimate $I_{\alpha}^{\prime}(z)$, use is made of the fact, [7, p. 79 (2)], that

$$
I_{\alpha}^{\prime}(z)=\frac{1}{2}\left[I_{\alpha-1}(z)+I_{\alpha+1}(z)\right] .
$$

Combining this with (2.8) and (2.9) then shows that

$$
I_{\alpha}^{\prime}(z)=\left(\frac{1}{2} z\right)^{\alpha-1} / 2 \Gamma(\alpha)+O\left(z^{\alpha+1}\right), \quad 0<z \leqq 1,
$$

and

$$
I_{\alpha}^{\prime}(z)=\frac{e^{z}}{\sqrt{ }(2 \pi z)}\left[1+O\left(z^{-1}\right)\right], \quad 1 \leqq z .
$$

To make the conjugate Poisson integral, $\tilde{f}(x, y)$, satisfy (1.4), it is clear that it should be defined by

$$
f(x, y)=\int_{0}^{1} q f(z) e^{-z} z^{\alpha} d z
$$

where

$$
q=\sqrt{ } y \int_{0}^{1} \frac{\partial}{\partial y}\left[\frac{\exp \left(\frac{x^{2}}{4 \log r}\right) \exp \left(\frac{-r(y+z)}{1-r}\right) I_{\alpha}\left(\frac{2 \sqrt{ }(r y z)}{1-r}\right)}{r(1-r) \sqrt{ }(-\log r)(r y z)^{\alpha / 2}}\right] d r
$$


Expression (2.13) is obtained from (2.2) by differentiating with respect to $y$, integrating with respect to $x$, formally interchanging operations, using the fact obtained from (1.3) that $q$ should approach 0 when $x$ approaches $\infty$, and multiplying by $-\sqrt{ } y$. The existence of the integral in (2.12) and the fact that $f(x, y)$ satisfies (1.4) are not obvious; these questions will be treated in $\S 3$.

The following definitions will be useful in the discussion of $q$ :

$$
\begin{aligned}
w & =\frac{\sqrt{ }(1-r)}{\pi^{1 / 2} \sqrt{ }(-\log r)} \exp \left(\frac{x^{2}}{4 \log r}\right), \\
s & =(\sqrt{ } y) \frac{\partial}{\partial y}\left[\frac{I_{\alpha}\left(\frac{2 \sqrt{ }(r y z)}{1-r}\right) \exp \left(\frac{-r(y+z)}{1-r}\right)}{r(1-r)^{3 / 2}(r y z)^{\alpha / 2}}\right], \\
b & =\exp \left(\frac{-r y+2 \sqrt{ }(r y z)-r z}{(1-r)}\right)
\end{aligned}
$$

and

$$
v=\frac{b(\sqrt{ } z-\sqrt{ }(r y))}{2 \pi^{1 / 2}(1-r)^{2}(\sqrt{ } r)(y z)^{\alpha / 2+1 / 4}}
$$

When $b$ is estimated the following alternate forms will be used:

$$
b=e^{y} \exp \left(-(\sqrt{ } y-\sqrt{ }(r z))^{2} /(1-r)\right)
$$

and

$$
b=e^{z} \exp \left(-(\sqrt{ } z-\sqrt{ }(r y))^{2} /(1-r)\right) .
$$

Finally, define $d$ as the value of $r$ in $(0,1)$ for which $2 \sqrt{ }(r y z) /(1-r)=1$.

Since $2 \sqrt{ }(r y z) /(1-r)$ is monotone increasing in $r$ for $0<r<1, d$ is greater than a value of $r$ that makes $2 \sqrt{ }(r y z) /(1-r)<1$ and less than a value of $r$ that makes $2 \sqrt{ }(r y z) /(1-r)>1$. This gives easily the facts that

$$
1 / 8 y z<d<1 / 4 y z, \quad y z \geqq 3 / 4
$$

and

$$
\max (1 / 8,1-2 \sqrt{ }(y z))<d<1-3 \sqrt{ }(y z) / 4, \quad 0<y z \leqq 5 / 4 .
$$

The value of $d$ is needed since the argument of the Bessel function in $q$ is $2 \sqrt{ }(r y z) /$ $(1-r)$, and the estimates to be used for the Bessel function depends on whether the argument is greater or less than 1.

With the given notation the kernel $q$ can be written as

$$
q=\int_{0}^{1} w s d r
$$


Performing the differentiation in the definition of $s$ and using (2.8)-(2.11) now shows that

$$
s=\frac{(\sqrt{ } y) \exp \left(\frac{-r(y+z)}{1-r}\right)}{(1-r)^{\alpha+5 / 2}} O\left(1+\frac{z}{1-r}\right), \quad 0<r \leqq d
$$

and

$$
s=v r^{-\alpha / 2-1 / 4}+\frac{b O\left(\frac{1}{\sqrt{ } z}+\frac{1}{\sqrt{ }(r y)}\right)}{(1-r)(\sqrt{ } r)(r y z)^{\alpha / 2+1 / 4}}, \quad d \leqq r<1 .
$$

3. Simpler facts about the conjugate Poisson integral. These are stated in the following theorem.

THEOREM 1. For a fixed $y>0, \alpha>-1$ and $a>0,|q|$ is bounded for $z>0$ and $x \geqq a$. If $f(y)$ is in $L^{1}\left(e^{-y} y^{\alpha}\right)$ on $[0, \infty)$, then $\tilde{f}(x, y)$ exists for every positive $x$ and $y$ and (1.2)-(1.4) are valid. If $f(y)$ has Laguerre expansion $\sum a_{n} L_{n}^{\alpha}(y)$, then for each $x>0$, $\tilde{f}(x, y) / \sqrt{ } y$ has the Laguerre expansion

$$
\sum_{n=1}^{\infty} \frac{-a_{n} \exp (-x \sqrt{ } n) L_{n-1}^{\alpha+1}(y)}{\sqrt{ } n}
$$

If $1 \leqq p<2$, there exists an $f(y)$ in $L^{p}\left(e^{-y} y^{\alpha}\right)$ such that for some positive $x$ (3.1) diverges for every $y$. If $x>0$ and $f(y)$ is in $L^{2}\left(e^{-y} y^{\alpha}\right)$, then (3.1) converges to $\tilde{f}(x, y) / \sqrt{ } y$ for almost every $y$.

This will be proved in the order that it is stated. Using (2.23) and (2.24) in (2.22) expresses $q$ as the sum of an integral from 0 to $d$ and one from $d$ to 1 . In the first of these integrals use the fact that $\exp (-r z /(1-r)) \leqq 1$; in the second use $(2.18)$ and the facts that $\sqrt{ } z-\sqrt{ }(r y) \leqq \sqrt{ } z+\sqrt{ }(r y)$ and $1 / \sqrt{ }(r y z) \leqq 2 /(1-r)$. Then using the fact that $(1-r) /-\log r \leqq 1$ shows that $|q|$ is bounded by a constant times the sum of

$$
(\sqrt{ } y) \int_{0}^{d}(1-r)^{-\alpha-5 / 2}\left(1+\frac{z}{1-r}\right) \exp \left(\frac{-r y}{1-r}\right) \exp \left(\frac{x^{2}}{4 \log r}\right) d r
$$

and

$$
e^{y} \int_{0}^{1} \frac{(\sqrt{ } z+\sqrt{ }(r y)) \exp \left(\frac{-(\sqrt{ } y-\sqrt{ }(r z))^{2}}{1-r}\right) \exp \left(\frac{x^{2}}{4 \log r}\right)}{\sqrt{ } r(1-r)^{2}(r y z)^{\alpha / 2+1 / 4}} d r
$$

By considering separately $0<r<\frac{1}{2}$ and $\frac{1}{2} \leqq r<1$ and replacing $-\log r$ by a suitable multiple of $1-r$ in the second case, it is clear that for a fixed $k>0$

$$
\exp \left(x^{2} / 4 \log r\right) \leqq C(1-r)^{k}\left(1+x^{-2 k}\right) .
$$

Using (3.4) with $k=2$ shows that $|q|$ is bounded by $C\left(1+x^{-4}\right)$ times the sum of

$$
(\sqrt{ } y) \int_{0}^{d}(1-r)^{-\alpha-1 / 2}\left(1+\frac{z}{1-r}\right) \exp \left(\frac{-r y}{1-r}\right) d r
$$


and

$$
e^{y} \int_{0}^{1} \frac{\exp \left(\frac{-(\sqrt{ } y-\sqrt{ }(r z))^{2}}{1-r}\right)(\sqrt{ } z+\sqrt{ }(r y))}{(\sqrt{ } r)(r y z)^{\alpha / 2+1 / 4}} d r .
$$

Since $1+x^{-4}$ is bounded for $x \geqq a$, proving that (3.5) and (3.6) have bounds independent of $z$ will complete the proof of the first assertion in Theorem 1 .

To treat (3.5) use (2.20). If $y z \geqq 1, d \leqq 1 / 4 y z$ and (3.5) has a bound independent of $z$. If $y z<1$, then $z<1 / y$ and the integrand has a bound independent of $z$.

To estimate (3.6) several cases will be considered. If $y z \geqq 1$ and $z \leqq 4 y$, there is a bound for (3.6) independent of $z$ since the integrand is a continuous function and $z$ lies in the compact interval [4y,1/y]. If $y z \geqq 1$ and $z>4 y,(2.20)$ shows that (3.6) is bounded by

$$
e^{y}\left|\int_{1 / 8 y z}^{4 y / z} 2 \sqrt{ }(z / r)\left(1+y^{-\alpha-1 / 2}\right) d r\right|+e^{y} \int_{4 y / z}^{1} \frac{e^{-r z / 2} 2 \sqrt{ } z}{(r y z)^{\alpha / 2+1 / 4} \sqrt{ } r} d r .
$$

Both of these integrals are easily seen to have bounds independent of $z$ by making the substitution $r z=u$. If $1 / 16 \leqq y z \leqq 1$ or if $y z \leqq 1 / 16$ and $z \geqq \frac{1}{4} y$, there is a bound for (3.6) independent of $z$ by continuity. Finally if $y z \leqq 1 / 16$ and $z<\frac{1}{4} y,(2.21)$ shows that (3.6) is bounded by

$$
C e^{y} \int_{1-2 \sqrt{ }(y z)}^{1} \frac{\exp \left(\frac{-y}{2(1-r)}\right) \sqrt{ } y}{(y z)^{\alpha / 2+1 / 4}} d r .
$$

Replacing $r$ by the lower limit of integration, it is clear that the integrand is bounded for $0<z<\frac{1}{4} y$.

The existence of $\tilde{f}(x, y)$ for $x>0$ follows immediately from the boundedness just proved for $q$.

To prove that the differential equations (1.2)-(1.4) are valid, it is first necessary to show that the kernels $\partial P / \partial x, \partial P / \partial y, \partial q / \partial x, \partial q / \partial y, \partial^{2} q / \partial x^{2}$ and $\partial^{2} q / \partial y^{2}$ can be obtained from the definitions of $P$ and $q,(2.2)$ and (2.13), by differentiating under the integral sign. In a suitable neighborhood of any positive $x, y$ and $z$ the differentiated integrands are all bounded by $C / r(1-\log r)^{3 / 2}$; this can be shown easily by using (2.8)-(2.11) and the equation given for $I_{\alpha}^{\prime}(z)$. This justifies the differentiation under the integral sign. Following the procedure just used on $q$ with a few changes in the details, all the kernels mentioned above can be shown to be bounded in $z$ for a fixed $y$ and $0<a \leqq x \leqq a^{-1}$. This justifies the computation of the various derivatives needed in (1.2)-(1.4) by differentiating under the integral sign in the definitions of $f(x, y)$ and $\tilde{f}(x, y),(2.1)$ and (2.12). The equalities (1.2)-(1.4) then follow from the fact that the kernels that arise are the same. In the case of (1.4) the equality of the kernels is immediate; for (1.2) and (1.3) an integration by parts must be performed to make this apparent. 
Starting with (2.7) and using (2.6) to justify the interchange of operations, it is clear that

$$
q=\sum_{n=0}^{\infty} \frac{-\exp (-x \sqrt{ } n)(\sqrt{ } y) L_{n-1}^{\alpha+1}(y) L_{n}^{\alpha}(z)}{(\sqrt{ } n) \Gamma(\alpha+1)\left(\begin{array}{c}
n+\alpha \\
n
\end{array}\right)}
$$

and

$$
\int_{0}^{\infty} q L_{n-1}^{\alpha+1}(y) e^{-y} y^{\alpha+1 / 2} d y=-(\sqrt{ } n) \exp (-x \sqrt{ } n) L_{n}^{\alpha}(z)
$$

for $x>0$.

To prove that (3.1) is the Laguerre expansion of $\tilde{f}(x, y) / \sqrt{ } y,(3.8)$ and Fubini's theorem will be used. To justify the use of Fubini's theorem, first, the estimate

$$
\int_{(1-r)^{2} / 4 r z}^{\infty} y^{a} \exp \left[\frac{-(\sqrt{ } y-\sqrt{ }(r z))^{2}}{1-r}\right] d y \leqq C+C z^{a+1}
$$

will be proved for a fixed $a>-1$. If $z<1$, replacing the lower limit of integration by 0 and the exponential term by $\exp \left[1-(\sqrt{ }(y)-1)^{2}\right]$, shows that the left side of (3.9) is bounded by a constant. If $z \geqq 1$ and $r<1 / 6 z$, then $(1-r)^{2} / 4 r z>4 r z$ so that the exponential can be replaced by $e^{-y / 4}$. The lower limit can then be replaced by 0 and the resulting integral is bounded. If $z \geqq 1$ and $r \geqq 1 / 6 z$, the integral is bounded by the sum of integrals of the same integrand over $[0,6 r z]$ and $[6 r z, \infty)$. In the first of these replace the exponential by 1 ; in the second replace it by $e^{-y / 4}$. Both parts then are bounded by $C z^{a+1}$. This completes the proof of (3.9).

Next, it will be shown that for a fixed $n$ that

$$
\int_{0}^{\infty}\left|q e^{-y} y^{\alpha+1 / 2} L_{n}^{\alpha+1}(y)\right| d y \leqq C\left(1+z^{C}\right)\left(1+x^{-C}\right) .
$$

To prove this, majorize $\left|L_{n}^{\alpha+1}(y)\right|$ by $C y^{n}+C$ and $|q|$ by the sum of (3.2) and (3.3). Replacing the upper limit of (3.2) by 1 and inverting the order of integration, the estimate (3.10) follows easily for this part. The part contributed by (3.3) is estimated by reversing the order of integration, replacing the $r y z$ term by $(1-r)^{2}$ if $\alpha>-\frac{1}{2}$ and using (3.9).

Now if $f(z)$ has a Laguerre expansion, $f(z) e^{-z} z^{\alpha+k}$ is integrable on $[0, \infty)$ for every nonnegative integer $k$. An inspection of (3.10) then shows that

$$
\left|e^{-z} z^{\alpha} f(z) q e^{-y} y^{\alpha+1 / 2} L_{n-1}^{\alpha+1}(y)\right|
$$

is integrable as a function of $y$ and $z$ if $x>0$. This allows the use of Fubini's theorem and (3.8) to obtain

$$
\int_{0}^{\infty} \tilde{f}(x, y) e^{-y} y^{\alpha+1 / 2} L_{n-1}^{\alpha+1}(y) d y=\frac{-a_{n}}{\sqrt{ } n} \exp (-x \sqrt{ } n) \Gamma(\alpha+2)\left(\frac{n+\alpha}{n-1}\right)
$$

provided that $x>0$. This is equivalent to the statement that (3.1) is the Laguerre expansion of $f(x, y) / \sqrt{ } y$ for $x>0$. 
The last two assertions are proved in the same way that the corresponding statements in [3, Lemma 4] were proved.

4. A basic lemma. The norm inequalities and the convergence theorems for $f(x, y)$ depend upon the following lemma.

LEMMA. For every $x \geqq 0, q$ can be written as the sum of two functions, $j(x, y, z)$ and $k(x, y, z) e^{z} z^{-\alpha}$, with the following properties. The function $|j(x, y, z)|$ is bounded by a constant times a function, $n(y, z)$, that is independent of $x$, is monotone increasing in $z$ for $z<y$ and is monotone decreasing in $z$ for $z>y$. Furthermore, $\int_{0}^{\infty} e^{-z} z^{\alpha} n(y, z) d z$ has $a$ bound independent of $y$. The function $k(x, y, z)=0$ if $|y-z| \geqq \min \left(\frac{1}{4}, \frac{1}{4} y\right)$, $k(x, y, y+h)=-k(x, y, y-h)$ and $(y-z) k(x, y, z)$ (defined as 0 for $y=z$ ) as a function of $z$ has total variation bounded by a constant independent of $x$ and $y$.

Let $K(x, y, z)$ be the odd part of the conjugate Poisson kernel for Hermite expansions for $z$ near $y$ as defined in (3.15) of [2]. Let $M$ denote $\min \left(\frac{1}{4} y, \frac{1}{4}\right)$. Then define the function $k$ as

$$
\begin{aligned}
k(x, y, z) & =\frac{K\left(\frac{1}{2} x, \sqrt{ } y, \sqrt{ } z\right)}{2 \sqrt{ } y}, & & y-M<z<y, \\
& =\frac{-K\left(\frac{1}{2} x, \sqrt{ } y, \sqrt{ }(2 y-z)\right)}{2 \sqrt{ } y}, & & y<z<y+M, \\
& =0, & & |y-z| \geqq M .
\end{aligned}
$$

That $k$ has the first two properties asserted in the lemma is immediate. To prove the third, observe that for $y-M<z<y$,

$$
(z-y) k(x, y, z)=\frac{\sqrt{ } z+\sqrt{ } y}{2 \sqrt{ } y}(\sqrt{ } y-\sqrt{ } z) K\left(\frac{1}{2} x, \sqrt{ } y, \sqrt{ } z\right)
$$

For this range of $z(\sqrt{ } z+\sqrt{ } y) / 2 \sqrt{ } y$ is uniformly bounded and has uniformly bounded variation by inspection. The term $(\sqrt{ } y-\sqrt{ } z) K\left(\frac{1}{2} x, \sqrt{ } y, \sqrt{ } z\right)$ is uniformly bounded and has uniformly bounded variation since, as shown in $\S 6$ of [2], $(y-z) K(x, y, z)$ has these properties. Therefore, $(z-y) k(x, y, z)$ has uniformly bounded variation for $z<y$ and by symmetry it has uniformly bounded variation for all $z$.

The function $j(x, y, z)$ must then be defined to be $q-e^{z} z^{-\alpha} k(x, y, z)$. It will be shown in $\S \S 5,6$ and 7 that if the constant $C$ is properly chosen, then $|j(x, y, z)|$ $\leqq C n(y, z)$ for $x \geqq 0$ where for $0<y \leqq 1$

$$
\begin{aligned}
n(y, z) & =y^{-\alpha-1}, & & 0<z \leqq 3 y / 4, \\
& =y^{-\alpha-1} \log (y /|y-z|), & & 3 y / 4<z \leqq 5 y / 4, \\
& =(\sqrt{ } y) z^{-\alpha-3 / 2}, & & 5 y / 4<z \leqq 2, \\
& =(\sqrt{ } y) 2^{-\alpha-2}, & & 2<z
\end{aligned}
$$


and for $y>1$

$$
\begin{aligned}
n(y, z) & =\frac{1}{\sqrt{ } y(2 \alpha+4)^{\alpha+1}}, & & 0<z \leqq \min \left(\alpha+2, \frac{3 y}{4}\right), \\
& =\frac{e^{z}}{\sqrt{ } y z^{\alpha+1 / 22^{\alpha+1}}}, & & \alpha+2<z \leqq \frac{3 y}{4}, \\
& =\frac{e^{z}}{y^{\alpha+1}}\left(1+\frac{y}{8(y-z)^{3 / 2}}\right), & & \frac{3 y}{4}<z \leqq y-\frac{1}{4}, \\
& =e^{y} y^{-\alpha}(1-\log |y-z|), & & y-\frac{1}{4}<z \leqq y+\frac{1}{4}, \\
& =e^{y} y^{-\alpha}, & & y+\frac{1}{4}<z .
\end{aligned}
$$

It is easy to verify that $n(y, z)$ has the asserted properties since $e^{z} z^{-\alpha-1 / 2}$ is monotone increasing for $z \geqq \alpha+\frac{1}{2}$.

All that remains to prove in the lemma is the inequality $|j(x, y, z)| \leqq C n(y, z)$. Since $k(x, y, z)$ is zero for $|y-z| \geqq \frac{1}{4} y$, this can be done by proving the following three inequalities:

$$
\begin{gathered}
|q| \leqq C n(y, z), \quad|y-z| \geqq \frac{1}{4} y, \\
\left|q-\int_{0}^{1} w v d r\right| \leqq C n(y, z), \quad|y-z|<\frac{1}{4} y, \\
\left|\int_{0}^{1} w v d r-k(x, y, z) e^{z} z^{-\alpha}\right| \leqq C n(y, z), \quad|y-z|<\frac{1}{4} y .
\end{gathered}
$$

These inequalities will be proved in $\S \S 5,6$ and 7 respectively.

5. Proof of (4.4). Using (2.22) and the fact that $|w(x, r)| \leqq 1$, it is sufficient to prove that

$$
\int_{0}^{1}|s| d r \leqq C n(y, z) \text { for }|y-z| \geqq \frac{1}{4} y .
$$

To use the estimates (2.23) and (2.24) it is necessary to prove separately the inequalities

$$
\int_{0}^{d}|s| d r \leqq C n(y, z), \quad|y-z| \geqq \frac{1}{4} y
$$

and

$$
\int_{d}^{1}|s| d r \leqq C n(y, z), \quad|y-z| \geqq \frac{1}{4} y .
$$

To prove (5.1), use (2.23). If $y>1$ and $z>1$, use (2.20), the fact obtained from (2.20) that $r<\frac{1}{4}$ and the fact that the exponential is bounded by 1 , to show that the integral is bounded by $C y^{-1 / 2}$. Otherwise, make the substitution $u=(y+z) /(1-r)$ and then change the upper limit to $\infty$. If $y+z \geqq 1$, use the fact that for $x \geqq 1$

$$
\int_{x}^{\infty} u^{a} e^{-u} d u \leqq C x^{a} e^{-x}
$$


where $C$ depends only on $a$. This shows that the integral is bounded by

$$
C(\sqrt{ } y)(1+z) /(y+z)
$$

in this case. If $y+z<1$, the integral is easily seen to be bounded by

$$
\mathrm{C} \sqrt{ } y /(y+z)^{\alpha+3 / 2} .
$$

In all of these cases (5.1) is true.

To prove (5.2), observe by use of (2.24) and (2.17) that for $|y-z| \geqq \frac{1}{4} y$ and $r \geqq d$,

$$
|s| \leqq \frac{C b \sqrt{ }(y z)}{(r y z)^{\alpha / 2+3 / 4}(1-r)^{2}}\left[(\sqrt{ } z-\sqrt{ }(r y))+\frac{1-r}{\sqrt{ } z}+\frac{1-r}{\sqrt{ }(r y)}\right]
$$

Four cases will be considered in proving (5.2): case A, $y z>1, z \leqq 3 y / 4$; case B, $y z>1, z \geqq 5 y / 4$; case $\mathrm{C}, y z \leqq 1, z \leqq 3 y / 4$; case $\mathrm{D}, y z \leqq 1, z \geqq 5 y / 4$. For case A the integral in (5.2) will be split into integrals over the intersection of $[d, 1]$ with $[0, z / 2 y],[z / 2 y, 5 z / 4 y],[5 z / 4 y, 15 / 16]$ and $[15 / 16,1]$. For case B the integral will be split into integrals over the intersection of $[d, 1]$ with $[0, y / 2 z],[y / 2 z, 6 y / 5 z]$, $[6 y / 5 z, 24 / 25]$ and $[24 / 25,1]$. For cases C and D the integral will not be split. These ten integrals to be considered will be called integrals 1 to 10 respectively.

For each of these ten integrals (5.4) will be used. Expressions that majorize the variable terms on the right side of (5.4) will now be given to replace those terms and make the ten integrals simple to estimate. The exponential of the following quantities will be used respectively as estimates for $b$ in the ten integrals:

$$
\begin{gathered}
z-C z, \quad z-\frac{C(z-r y)^{2}}{z}, \quad z-C r y, \quad z-\frac{C y}{1-r}, \quad y-C y, \\
y, \quad y-C r z, \quad y-\frac{C z}{1-r}, \quad C-\frac{C y}{1-r} \text { and } C-\frac{C z}{1-r} .
\end{gathered}
$$

The first eight of these are easily obtained from (2.18) or (2.19). The ninth is obvious if $r>.9$ or $y>5$, and the $C$ 's can be adjusted to make it true if $r \leqq .9$ and $y \leqq 5$. The tenth is like the ninth. Replace the long expression in brackets in (5.4) by $C \sqrt{ } y$ in integrals 4 and 9 , by $(C|z-r y|+C) / \sqrt{ } z$ in 2 , by $C \sqrt{ }(r y)$ in 3 and by $C \sqrt{ } z$ in the rest. For these and the next set of estimates considerable use is made of the fact that $r \geqq d$ so that $2 \sqrt{ }(r y z) /(1-r) \geqq 1$. Replace the ryz term in the denominator by $C z^{2}$ in 2, by $C y z$ in 9 and 10, by $C y^{2}$ in 6 and by $C$ in the others. Leave the $1-r$ term unchanged in integrals $4,8,9$ and 10 and replace it by $C$ in the others.

By performing the integrations and considering appropriate cases, integrals 1,4 , $5,7,8,9$ and 10 are seen to be bounded by $C n(y, z)$. If integral 2 has a nonempty interval of integration, $d \leqq 5 z / 4 y$, and (2.20) shows that $z$ is bounded below. Making the substitution $u=|z-r y| / \sqrt{ } z$ and using (5.3) will give the estimate for integral 2. For integral 3, note that by (2.20) the lower limit is greater than $1 / 8 y z$ if $z$ is small, substitute $u=r y$ and use (5.3). For integral $6, y$ is bounded below as $z$ was in 2 . 
With these suggestions it is easy to complete the proof of (5.2) and, therefore, of (4.4).

6. Proof of (4.5). The approach here is similar to that in $\S 5$. Again, since $|w(x, r)| \leqq 1$, it is sufficient to prove that $\int_{0}^{1}|s-v| d r \leqq C n(y, z)$ for $|y-z|<\frac{1}{4} y$. The difference between (2.23) and (2.24) requires that the integral be split up and the form of (2.24) requires that the second part be rewritten. Consequently, (4.5) will be proved by proving that for $|y-z|<\frac{1}{4} y$

$$
\int_{0}^{d}(|s|+|v|) d r \leqq C n(y, z)
$$

and

$$
\int_{d}^{1}\left(\left|s-v r^{-\alpha / 2-1 / 4}\right|+|v|\left|r^{-\alpha / 2-1 / 4}-1\right|\right) d r \leqq C n(y, z) .
$$

To prove (6.1), observe that the proof of (5.1) is valid for the first part of (6.1). This reduces the proof of $(6.1)$ to the proof that

$$
\int_{0}^{d}|v| d r \leqq C n(y, z), \quad|y-z|<\frac{1}{4} y
$$

If $y>1$, use (2.17), replace $b$ by $e^{z}$ if $z<y$ or by $e^{y}$ if $z>y$, replace $\sqrt{ } z-\sqrt{ }(r y)$ by $C \sqrt{ } y$ and $1-r$ by $C$. Using the fact that $y z>3 / 4,(2.20)$ shows that $d \leqq 1 / 4 y z$ and the left side of (6.3) is bounded by $C y^{-\alpha-1} e^{z}$ if $z<y$ and by $C y^{-\alpha-1} e^{y}$ if $z>y$. This is sufficient to prove (6.3) for $y>1$. If $y \leqq 1$, replace $b$ by 1 and $\sqrt{ } z-\sqrt{ }(r y)$ by $C \sqrt{ } y$. Since $y z<5 / 4,(2.21)$ shows that $d<1-3 \sqrt{ }(y z) / 4$ and the integral is easily seen to be less than $C y^{-\alpha-1}$. This completes the proof of (6.3) and, therefore, of (6.1).

Making use of the fact that for a fixed $a\left|1-r^{a}\right| \leqq C\left(1+r^{a}\right)(1-r)$, the fact that $|y-z|<\frac{1}{4} y,(2.17)$ and (2.24), the integrand in (6.2) is found to be bounded by

$$
\frac{\operatorname{Cby}\left[(r y)^{-1 / 2}+\left(1+r^{\alpha / 2+1 / 4}\right)|\sqrt{ } z-\sqrt{ }(r y)|\right]}{(1-r)(r y z)^{\alpha / 2+3 / 4}} .
$$

Three cases will be considered in proving (6.2): case A, $y>1,3 y / 4<z<y$; case $\mathrm{B}, y>1, y<z<5 y / 4$; case $\mathrm{C}, y \leqq 1$. For case A the integral in (6.2) will be split into integrals over

$$
[d, 1 / 3], \quad[1 / 3,(3 z-y) / 2 y], \quad[(3 z-y) / 2 y,(y+z) / 2 y] \text { and } \quad[(y+z) / 2 y, 1] \text {. }
$$

For case $\mathrm{B}$ the integral will be split into integrals over $\left[d, \frac{1}{4}\right],\left[\frac{1}{4},(3 y-z) / 2 z\right]$, $[(3 y-z) / 2 z,(y+z) / 2 z]$, and $[(y+z) / 2 z, 1]$. For case $\mathrm{C}$ the integral will not be split. These nine integrals to be considered will be called integrals 1 to 9 respectively. As in the proof of (5.2), replacements for the various terms in (6.4) will be given for each of these integrals. 
The replacements for $b$ are not quite so obvious in this case as they were in the proof of (5.2). For the first four integrals use the fact obtained from (2.19) that $b \leqq e^{z} \exp \left(-C(z-r y)^{2} / y(1-r)\right)$. Now $z-r y=(z-y)+y(1-r)$. In the first and second integrals $y(1-r) \geqq 3|z-y| / 2$ so that $(z-r y)^{2} \geqq C y^{2}(1-r)^{2}$ while in the fourth integral $|z-y| \geqq 2 y(1-r)$ and $(z-r y)^{2} \geqq C(y-z)^{2}$. Therefore, $b$ can be replaced by the exponential of $z-C y, z-C y(1-r)$ and $z-C(y-z)^{2} / y(1-r)$ in the first, second and fourth integrals respectively. For the third, since $y-z \geqq$ $2 y(1-r) / 3, b$ can be replaced by $e^{z} \exp \left(-C(z-r y)^{2} /(y-z)\right)$. The same reasoning permits the replacement of $b$ in the fifth through eighth integrals respectively by the exponential of $y-C z, y-C z(1-r), y$ and $y-C(z-y)^{2} / z(1-r)$. For the ninth integral replace $b$ by $\exp \left(C-C(y-z)^{2} / y(1-r)\right)$; if $y(1-r)<2|y-z|$, this follows from the previous reasoning, while if $y(1-r) \geqq 2|y-z|$, adjusting the first $C$ will make it valid.

The term in brackets in the numerator of (6.4) can be replaced in the integrals respectively by $C$ times

$$
\begin{aligned}
& y^{1 / 2}+y^{-\alpha}, \quad y^{-1 / 2}+y^{1 / 2}(1-r), \quad y^{-1 / 2}(1+|z-r y|), \quad y^{-1 / 2}(1+y-z), \\
& y^{1 / 2}+y^{-\alpha}, \quad y^{-1 / 2}+y^{1 / 2}(1-r), \quad y^{1 / 2}, \quad y^{-1 / 2}(1+z-y) \quad \text { and } y^{-1 / 2} \text {. }
\end{aligned}
$$

The term $r y z$ in the denominator of (6.4) should be replaced by $C$ for the first and fifth integrals and by $C y^{2}$ for the rest. Furthermore, $1-r$ should be replaced by $C$ in the first and fifth integrals and by $C|y-z| / y$ in the third and seventh integrals. For those integrals that have a variable exponential term let that exponent be the new variable in a change of variables. With this it is easy to show that all nine integrals are bounded by $C n(y, z)$. This completes the proof of (4.5).

7. Proof of (4.6). By making the change of variable $r=s^{2}$ it is immediate that

$$
\int_{0}^{1} w v d r=\frac{1}{2} Q\left(\frac{1}{2} x, \sqrt{ } y, \sqrt{ } z\right)(y z)^{-\alpha / 2-1 / 4}
$$

where $Q$ is the conjugate Poisson kernel for Hermite polynomials defined in (3.5) of [2]. Following the notation of [2] with $J(x, y, z)=Q(x, y, z)-K(x, y, z) e^{z^{2}}$ and using (7.1), the left side of (4.6) is seen to be bounded by the sum of

$$
\begin{gathered}
\frac{1}{2}\left|J\left(\frac{1}{2} x, \sqrt{ } y, \sqrt{ } z\right)\right|(y z)^{-\alpha / 2-1 / 4}, \\
\frac{1}{2}\left|K\left(\frac{1}{2} x, \sqrt{ } y, \sqrt{ } z\right)\right| e^{z}\left|(y z)^{-\alpha / 2-1 / 4}-z^{-\alpha} y^{-1 / 2}\right|
\end{gathered}
$$

and

$$
\left|\frac{1}{2} y^{-1 / 2} K\left(\frac{1}{2} x, \sqrt{ } y, \sqrt{ } z\right)-k(x, y, z)\right| e^{z} z^{-\alpha} .
$$

Proving that each of these is bounded by $\operatorname{Cn}(y, z)$ for $|y-z|<\frac{1}{4} y$ will complete the proof of (4.6).

That (7.2) is bounded by $C n(y, z)$ for $|y-z|<\frac{1}{4} y$ is immediate from the fact that $J(x, y, z)$ is bounded by the function $L(y, z)$ defined in (3.13) and (3.14) of [2]. 
By Lemma 4 of [2], $(z-y) K(x, y, z)$ has uniformly bounded variation. Therefore,

$$
|K(x, y, z)| \leqq C /|z-y| .
$$

Consequently,

$$
\frac{1}{2} y^{-1 / 2}\left|K\left(\frac{1}{2} x, \sqrt{ } y, \sqrt{ } z\right)\right| \leqq C /|z-y|, \quad|y-z|<\frac{1}{4} y .
$$

Now the expression, $\left|(y z)^{-\alpha / 2-1 / 4}-z^{-\alpha} y^{-1 / 2}\right|$, in (7.3) is bounded by $C|y-z| y^{-\alpha-3 / 2}$ for $|y-z|<\frac{1}{4} y$. Combining this fact with (7.6) shows that (7.3) is bounded by $e^{z} y^{-\alpha-1}$ for $|\sqrt{ } y-\sqrt{ } z|<\min \left(1, y^{-1 / 2}\right)$ and is zero elsewhere. This is sufficient to conclude that (7.3) is also bounded by $\operatorname{Cn}(y, z)$.

To treat (7.4) it is necessary to obtain several preliminary estimates. The first of these is the fact that

$$
\frac{|y-z|}{1-t^{2}} \exp \left(\frac{-(t y-z)^{2}}{1-t^{2}}\right) \leqq \frac{C}{|y-z|}
$$

for $|y-z|<1 / y$ and $0 \leqq t \leqq 1$. Using the fact that $u e^{-u}<1$ for $u$ positive, the left side of (7.7) is bounded by $|y-z| /(t y-z)^{2}$. If $z \geqq y$ this is sufficient for the proof. If $z<y$ and $t>(y+z) / 2 y$, this expression is also sufficient for the proof since for this case $(t y-z)>\frac{1}{2}(y-z)$. If $z<y$ and $t \leqq(y+z) / 2 y$, then $|y-z| /\left(1-t^{2}\right) \leqq y$, and this is sufficient.

The next inequality needed is

$$
\left|\frac{\partial}{\partial z}\left[(y-z) Q(x, y, z) e^{-z^{2}}\right]\right| \leqq \frac{C}{|y-z|}, \quad|y-z|<\min \left(1, y^{-1}\right) .
$$

By $\S 6$ of [2], the left side of (7.8) is bounded by expression (6.5) of [2]. The first term of (6.5) of [2] clearly satisfies the desired inequality and the integral term does also by use of Lemma 6 of [2] and (7.7).

Using (7.8) and the definition of $K$,

$$
\left|\frac{\partial}{\partial z}[(y-z) K(x, y, z)]\right| \leqq \frac{C}{|y-z|}, \quad|y-z|<\min \left(1, y^{-1}\right) .
$$

Combining this with (7.5) then gives

$$
\left|K_{3}(x, y, z)\right| \leqq C /(y-z)^{2}, \quad|y-z|<\min \left(1, y^{-1}\right) .
$$

Now the proof of (7.4) is simple. If $3 \leqq|y-z|<\frac{1}{4} y, K\left(\frac{1}{2} x, \sqrt{ } y, \sqrt{ } z\right)$ is 0 since $|\sqrt{ } y-\sqrt{ } z|>\min \left(1, y^{-1 / 2}\right)$ and $k$ is 0 since $|y-z|>\min \left(\frac{1}{4}, \frac{1}{4} y\right)$. Therefore, (7.4) is bounded by $C n(y, z)$ in this case. If $\frac{1}{4} \leqq|y-z|<3, k$ is 0 , and using (7.6) it is again clear that (7.4) is bounded by $C n(y, z)$. If $y-\min \left(\frac{1}{4}, \frac{1}{4} y\right)<z<y$, then by the definition of $k,(7.4)$ is zero. If $y<z<y+\min \left(\frac{1}{4}, \frac{1}{4} y\right)$, then the definition of $k$ and the fact that $K(x, y, z)=-K(x, y, 2 y-z)$ show that $(7.4)$ is equal to

$$
\frac{1}{2} e^{z} z^{-\alpha} y^{-1 / 2}\left|K\left(\frac{1}{2} x, \sqrt{ } y, \sqrt{ } z\right)-K\left(\frac{1}{2} x, \sqrt{ } y, 2 \sqrt{ } y-\sqrt{ }(2 y-z)\right)\right| .
$$


It is easy to verify that $|y-z|<\min \left(\frac{1}{4}, \frac{1}{4} y\right)$ implies that $|\sqrt{ } z-\sqrt{ } y|$ and

$$
|(2 \sqrt{ } y-\sqrt{ }(2 y-z))-\sqrt{ } y|
$$

are less than $\min \left(1, y^{-1 / 2}\right)$. Consequently, the law of the mean can be applied to (7.11) using the estimate (7.10) for the derivative. Now

$$
(2 \sqrt{ } y-\sqrt{ }(2 y-z))-\sqrt{ } z=\frac{2(\sqrt{ } y-\sqrt{ } z)^{2}}{2 \sqrt{ } y+\sqrt{ }(2 y-z)-\sqrt{ } z} .
$$

This shows that the value of $K_{3}\left(\frac{1}{2} x, \sqrt{ } y, \sqrt{ } z\right)$ can be used in estimating (7.11) since $\sqrt{ } z$ is less than $2 \sqrt{ } y-\sqrt{ }(2 y-z)$. Furthermore, the right side of (7.12) is easily seen to be bounded by $C(y-z)^{2} y^{-3 / 2}$. Combining all these facts shows that (7.11) is bounded by $C e^{z} y^{-\alpha-1}$ for $y<z<y+\min \left(\frac{1}{4}, \frac{1}{4} y\right)$. This completes the proof that (7.4) is bounded by $C n(y, z)$, and, therefore, completes the proof of (4.6).

8. Conjugate function theorems. The principal results concerning the conjugate Poisson integral, $f(x, y)$, and its limit as $x \rightarrow 0^{+}, f(y)$, can now be stated.

THEOREM 2. Let $f(y)$ be in $L^{1}\left(e^{-y} y^{\alpha}\right), \alpha>-1$, on $[0, \infty)$, and for a fixed $y$ let $M$ denote $\min \left(\frac{1}{4}, \frac{1}{4} y\right)$. Define

$$
f^{*}(y)=\sup _{u \neq y} \frac{\int_{y}^{u}|f(z)| e^{-z} z^{\alpha} d z}{\int_{y}^{u} e^{-z} z^{\alpha} d z}
$$

and let $E_{a}=\left\{y\left|\sup _{x>0}\right| \tilde{f}(x, y) \mid>a\right\}$. Then there exists a constant, $C$, independent of $f$ such that

(a) $|\tilde{f}(x, y)| \leqq C\left(\sup _{0<\beta<\gamma<M}\left|\int_{\beta \leqq|z| \leqq \gamma} \frac{f(y-z)}{z} d z\right|+f^{*}(y)\right)$ for $x>0$,

$$
\lim _{\varepsilon \rightarrow 0^{+}} \int_{|y-z|>\varepsilon} f(z) q(0, y, z) e^{-z} z^{\alpha} d z \quad \text { exists for almost every } y,
$$

$$
\text { the limits in (c) and (d) are equal for almost every } y .
$$

THeOREM 3. Let $f(y)$ be in $L^{p}\left(e^{-y} y^{\alpha}\right), 1<p<\infty, \alpha>-1$ on $[0, \infty)$. Let $x$ be greater than 0 and let $P(x, y, z)$ be the Poisson kernel defined in (2.2). Then there exists constants, $A_{p}$, depending only on $p$ such that

$$
\lim _{x \rightarrow 0^{+}}\|\tilde{f}(x, y)-\tilde{f}(y)\|_{p}=0,
$$

$$
\|\tilde{f}(y)\|_{p} \leqq A_{p}\|f\|_{p}
$$

(c)

$$
f(x, y)=\int_{-\infty}^{\infty} \tilde{f}(z) P(x, y, z) e^{-z} z^{\alpha} d z \quad \text { for almost every } y \text {, }
$$




$$
\begin{gathered}
\left\|\sup _{x>0}|\tilde{f}(x, y)|\right\|_{p} \leqq A_{p}\|\tilde{f}(y)\|_{p}, \\
\|\tilde{f}(x, y)\|_{p} \leqq\left\|\tilde{f}(y)_{p}\right\| \text { for all } x>0,
\end{gathered}
$$

(f) if $f(y)$ has Laguerre expansion $\sum a_{n} L_{n}^{\alpha}(y)$, then $y^{-1 / 2} f(y)$ has Laguerre expansion

$$
\sum-n^{-1 / 2} a_{n} L_{n-1}^{\alpha+1}(y)
$$

THEOREM 4. If $\int_{-\infty}^{\infty}|f(y)|\left(\log ^{+}|f(y)|\right) e^{-y} y^{\alpha} d y=A$ is finite, then there exists a constant $C$, independent of $f$, such that

$$
\begin{gathered}
\lim _{x \rightarrow 0^{+}}\|\tilde{f}(x, y)-\tilde{f}(y)\|_{1}=0, \\
\|\tilde{f}(y)\|_{1} \leqq C(1+A), \\
\tilde{f}(x, y)=\int_{-\infty}^{\infty} \tilde{f}(z) P(x, y, z) e^{-z} z^{\alpha} d z \quad \text { for almost every } y, \\
\left\|\sup _{x>0} \tilde{f}(x, y)\right\|_{1} \leqq C(1+A), \\
\|\tilde{f}(x, y)\|_{1} \leqq\|\tilde{f}(y)\|_{1} \text { for } x>0 .
\end{gathered}
$$

These three theorems are proved in the same way that Theorems 2, 3 and 4 of [2] were proved. The lemma of this paper is used in place of Lemma 4 of [2] and facts from [3] about Laguerre series are used instead of the facts used in [2] about Hermite series. The only real difference occurs in justifying the use of Lemmas 2 and 3 of [2] since a suitable partition of $(0, \infty)$ with property $A$ for the weight function $e^{-y} y^{\alpha}$ is needed. For this, use the intervals $\left[2^{-n-1}, 2^{-n}\right], n=0,1,2, \ldots$ and $[n, n+1], n=1,2, \ldots$. These clearly have the three required properties for property $A$, and if $x$ is in one of these intervals, $\min \left(\frac{1}{4} x, \frac{1}{4}\right)$ is less than or equal to half the length of the interval. Slight modifications in the rest of the proofs of Theorems 2, 3 and 4 in [2] now complete the proof of the theorems of this section.

\section{REFERENCES}

1. A. P. Calderon and A. Zygmund, On the existence of certain singular integrals, Acta Math. 88 (1952), 85-139. MR 14, 637.

2. B. Muckenhoupt, Hermite conjugate expansions, Trans. Amer. Math. Soc. 139 (1969), 243-260.

3. - Poisson integrals for Hermite and Laguerre expansions, Trans. Amer. Math. Soc. 139 (1969), 231-242.

4. B. Muckenhoupt and E. M. Stein, Classical expansions and their relation to conjugate harmonic functions, Trans. Amer. Math. Soc. 118 (1965), 17-92. MR 33 \#7779.

5. E. M. Stein, On limits of sequences of operators, Ann. of Math. (2) 74 (1961), 140-170. MR 23 \#A2695. 
6. G. Szegö, Orthogonal polynomials, rev. ed., Amer. Math. Soc. Colloq. Publ., vol. 23, Amer. Math. Soc., Providence, R. I., 1959. MR 21 \#5029.

7. G. N. Watson, Theory of Bessel functions, Cambridge Univ. Press, Cambridge, 1966.

8. A. Zygmund, Trigonometric series, Vols. I, II, 2nd rev. ed., Cambridge Univ. Press, New York, 1959. MR 21 \#6498.

\section{Rutgers, The State University,} NeW BRunswick, NeW JerSeY 